\title{
Live Demonstration: A Portable Microsensor Fusion System with Real-Time Measurement for On-Site Beverage Tasting
}

\author{
Josep Maria Margarit-Taulé1,2, Pablo Giménez-Gómez ${ }^{2}$, Roger Escudé-Pujol ${ }^{2}$, Manuel Gutiérrez-Capitán², \\ Cecilia Jiménez-Jorquera ${ }^{2}$ and Shih-Chii Liu ${ }^{1}$ \\ ${ }^{1}$ Institute of Neuroinformatics, University of Zurich and ETH Zurich, Zurich, Switzerland \\ ${ }^{2}$ Instituto de Microelectrónica de Barcelona, IMB-CNM(CSIC), Spain \\ $<$ josep.margarit@ini.uzh.ch>
}

\begin{abstract}
We demonstrate a portable multisensor fusion system for the automated analysis of multiple beverages. The system makes use of compact and low-power-consumption electronic equipment to simultaneously read out an array of microsensors formed by six ion-selective field-effect transistors (ISFETs), one conductivity sensor, one redox potential sensor, and two amperometric microelectrodes. A custom Python application running on a laptop computer receives real-time multivariate data via USB, and provides chemometric models to classify different varieties and to quantify relevant parameters of mineral water and wine. The software also includes a graphical user interface (GUI) to visualize readouts and analytical estimates.
\end{abstract}

\section{INTRODUCTION}

Increasingly strict process quality standards in food industry have promoted the development of rapid and precise analytical systems for monitoring the physico-chemical properties of products in their manufacturing cycle. In order to obtain such information in situ and in real time, portable and automatic systems for decentralized analysis are highly valuable. In this scenario, the use of chemical multisensor arrays as "electronic tongues" stand out due to their capability to generate multivariate data from liquid samples, enlarging the number of parameters that can be determined simultaneously. Among sensor alternatives, microelectrodes and ISFETs fabricated in semiconductor technologies present advantages such as miniaturization, robustness, high reproducibility, mass fabrication, and ease of integration with readout electronic circuitry, making them particularly suitable for incorporation into arrays for on-site measurements.

Multisensor data fusion allows to exploit cross-sensitivity, and uncover linear [1] and non-linear [2] structure in multisensor platforms. By using such methods, we demonstrate a portable, inexpensive electrochemical system that combines amperometric, conductimetric and potentiometric data from solid-state microtransducers to accurately perform nondestructive classification, discrimination, and quantitative analyses on multiple complex beverage products [3].

\section{DEMONSTRATION SETUP}

The demonstration setup is shown in Fig. 1. It employs two compact low-power multisensor meters [3], [4] to read out an array of microdevices formed by one conductivity sensor, one redox potential sensor, and two amperometric gold microelectrodes, together with six ISFETs sensitive to $\mathrm{pH}$,

This work has received funding from the European Union's Horizon 2020 research and innovation program under the Marie Sklodowska-Curie grant agreement No 747848, and from the Spanish national research and innovation program (MINECO, ref. TEC2014-54449-C3-1-R).

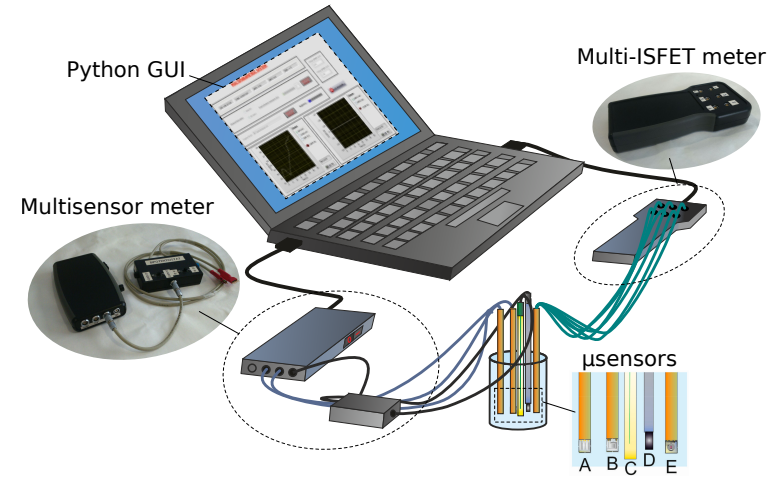

Figure 1. Demonstration setup for on-site beverage tasting. In detail: Conductivity/ORP sensor (A); amperometric sensor (B); reference electrode (C); counter electrode (D); and ISFET (E).

$\mathrm{Na}^{+}, \mathrm{K}^{+}, \mathrm{Ca}^{2+}, \mathrm{Cl}^{-}$, and $\mathrm{CO}_{3}{ }^{2-}$. All sensors are simultaneously immersed in the beverage under test. Multivariate data from both meters is transmitted to a laptop computer through the USB protocol, where chemical properties are modeled using linear regression and discriminant analysis on partial least squares scores, and non-linear networks running under Python. As a result, the system accurately quantifies relevant molecular/ion concentrations, and discriminates between different types of drinks and commercial brands, while assessing quality parameters such as taste, aging, and adulterants.

\section{VISITOR EXPERIENCE}

Visitors will choose which beverages to analyze among several samples of drinking water and wines. A Python GUI will enable them to visualize all sensor data on the fly, and to explore quantitative and qualitative estimates.

\section{REFERENCES}

[1] L. Moreno, A. Merlos, N. Abramova, C. Jiménez, and A. Bratov, "Multisensor array used as an "electronic tongue" for mineral water analysis," Sensors and Actuators B: Chemical, vol. 116, no. 1-2, pp. 130-134, Jul 2006. [Online]. Available: https://doi.org/10.1016/j.snb.2005.12.063

[2] G. Indiveri and S. Liu, "Memory and information processing in neuromorphic systems," Proc. IEEE, vol. 103, no. 8, pp. 1379-1397, Aug 2015. [Online]. Available: https://doi.org/10.1109/JPROC.2015.2444094

[3] P. Giménez-Gómez, R. Escudé-Pujol, F. Capdevila, A. Puig-Pujol, C. Jiménez-Jorquera, and M. Gutiérrez-Capitán, "Portable electronic tongue based on microsensors for the analysis of cava wines," Sensors, vol. 16, no. 11, p. 1796, Oct 2016. [Online]. Available: https://doi.org/10.3390/s16111796

[4] P. Giménez-Gomez, R. Escudé-Pujol, C. Jiménez-Jorquera, and M. Gutiérrez-Capitán, "Multisensor Portable Meter for Environmental Applications," IEEE Sensors J., vol. 15, no. 11, pp. 6517-6523, Nov 2015. [Online]. Available: https://doi.org/10.1109/JSEN.2015.2460011 